Classification

Physics Abstracts

61.16

\title{
Scanning tunneling microscopy of monoclonal immunoglobulin G
}

Jean Thimonier $\left({ }^{1,2}\right)$, Jean-Paul Chauvin $\left({ }^{1,3}\right)$, Jacques Barbet $\left({ }^{1,2}\right)$ and Jose Rocca-Serra $\left({ }^{1,2}\right)^{*}$

( $\left.{ }^{1}\right)$ CNRS GDR 976, Parc Scientifique et Technologique de Luminy, Case 901, 13288 Marseille Cedex 9, France

${ }^{2}$ ) Centre d'Immunologie INSERM-CNRS de Marseille-Luminy (UMR 4), Parc Scientifique et Technologique de Luminy Case 906, 13288 Marseille Cedex 9, France

${ }^{3}$ ) Laboratoire de Génétique et de Physiologie du Développement, (CNRS-Université Aix-Marseille II UMR 9943), Parc Scientifique et Technologique de Luminy Case 901, 13288 Marseille Cedex 9, France

(Received July 4; accepted December 16, 1994)

Résumé. - Une IgG 1 monoclonale de souris a été étudiée par microscopie par effet tunnel. La solution d'immunoglobuline a été déposée sur du mica fraîchement clivé, séchée, puis recouverte de platine et de carbone par évaporation rotative. Plusieurs images d'IgG ont été obtenues. Les formes générales (taille approximative, symétrie) sont restées apparentes sous le film métallique. Imager des IgG non recouvertes a été plus difficile. Plusieurs images d'IgG déposées sur graphite ont cependant été obtenues. Elles présentent une structure comparable à la structure attendue. Une nette amélioration de la qualité des images a été observée quand le courant tunnel a été réduit de 50 à 30 puis à $4 \mathrm{pA}$. Un bref traitement du graphite par ionisation a été essayé pour améliorer l'adsorption des molécules hydrophiles d'IgG. Cette technique peut être utile mais elle transforme la surface du graphite plane à l'échelle atomique en grains qui rendent difficile le repérage des molécules déposées.

\begin{abstract}
A mouse monoclonal $\mathrm{IgG}_{1}$ was studied by scanning tunneling microscopy. The immunoglobulin solution was deposited onto freshly cleaved mica, dried and rotary shadowed with platinum and carbon. Several images of IgG molecules were obtained. The overall features (approximate size, symmetry) remained apparent under the metal coating film. Imaging uncoated IgG was more difficult. However s veral images of IgG deposited on graphite consistent with the known structure were obtained. A definite improvement in image quality was observed when the tunneling current was reduced from 50, to 30 and $4 \mathrm{pA}$. Short treatment of graphite by glow discharge was tested to improve adsorption of the hydrophilic IgG molecules. The technique may be useful but transforms the atomically flat graphite surface into carbon grains which make difficult the localization of the deposited molecules.
\end{abstract}

* To whom correspondence should be addressed. 


\section{Introduction.}

Until recently, two major techniques have been used to study the structure of biological molecules: transmission electron microscopy (TEM) and X-ray diffraction crystallography. TEM requires the staining or shadowing of the molecules which are observed in vacuum. Resolution is limited but sample preparation techniques have been worked out for many years. X-ray crystallography is indeed the method of reference but solving the structure of a large protein may take a long time and is always dependent upon crystallization. Scanning tunneling microscopy has now provided physicists with structural information at the atomic level for crystalline solids $[1,2]$ and is proposed as a new tool for structural analysis in biology. Several reports have shown promising images of DNA [3, 4], polynucleotides [5], proteins [6-15]. Indeed, the scanning tunneling microscope (STM) is capable of extremely high resolution and it should be able to image the surface of a single molecule simply deposited on a conducting substratum. The adsorption of the molecule into a solid substrate, susceptible to induce structural perturbations, is still required. Only $\mathrm{Nu}$ clear Magnetic Resonance provides structural information in solution but the size of molecules amenable to this technique is rather small (a few tens of kilodaltons).

The immunoglobulin super-family is a large group of proteins whose structure comprises one or several domains of the immunoglobulin domain type [16]. Members of this family (T-cell receptors, MHC antigens, Thy-1,...) are involved in the regulation of important physiological functions through receptor-ligand and cell-cell interactions. We decided to use the STM to study the prototype, the immunoglobulin $\mathrm{G}$ molecule, which is also the most readily available and best characterized protein of this family.

\section{Materials and methods.}

The mouse monoclonal $\operatorname{IgG}_{1}$ ( $\gamma 1$ heavy chain, $\lambda$ light chain) was kindly provided by Immunotech S.A. (Marseille, France). The protein was purified from ascites fluids by Protein A-Sepharose (Pharmacia, Uppsala, Sweden) immunopurification followed by gel filtration on Superdex 200 (Pharmacia, Uppsala, Sweden). Molecular homogeneity was tested by analytical chromatography on Superdex 200 and sodium dodecyl sulfate polyacrylamide gel electrophoresis. The immunoglobulin was diluted to $15-50 \mu \mathrm{g} / \mathrm{ml}$ in water or in $0.2 \mathrm{M}$ ammonium acetate (pH 6) and centrifuged for $20 \mathrm{~min}$ at 20 psi (approximately 120,000 g) in a Beckman Airfuge just before use to pellet aggregated materials. Protein samples were deposited as a droplet on freshly cleaved highly oriented pyrolytic graphite (HOPG, Le Carbone Loraine, Paris, France) or mica. Samples were dried overnight on the bench or under vacuum. Imaging was performed directly on HOPG, or on mica after rotary shadowing at right angle with platinum and carbon $(80 \%-20 \%)$. Shadowing was performed using a Balzers BAF 300 apparatus (Balzers, Liechtenstein) with the sample maintained at $-140{ }^{\circ} \mathrm{C}$. The $\mathrm{Pt} / \mathrm{C}$ film thickness was set to $8 \AA$, as mesured by a quartz crystal monitor.

The STM was built in the laboratory by Humbert and his colleagues and designed for routine operation in air at room temperature [17]. Imaging was done with a tunneling current set between 4 and $50 \mathrm{pA}$ with the tip set between -600 and $-200 \mathrm{mV}$. Scan speed was varied between 0.2 and $2.0 \mu \mathrm{m} / \mathrm{s}$. Images were recorded as two dimensional arrays of 16 bit numbers and processed on an Atari TT computer with software developed in the laboratory.

Electron microscopy studies were performed on carbon replicas for samples deposited on mica or by pealing the first layers of carbon for samples deposited on HOPG by gluing with Araldite an electron microscope grid on the sample surface. The electron microscope used was a Philips EM300 (Eindoven, The Netherlands). 

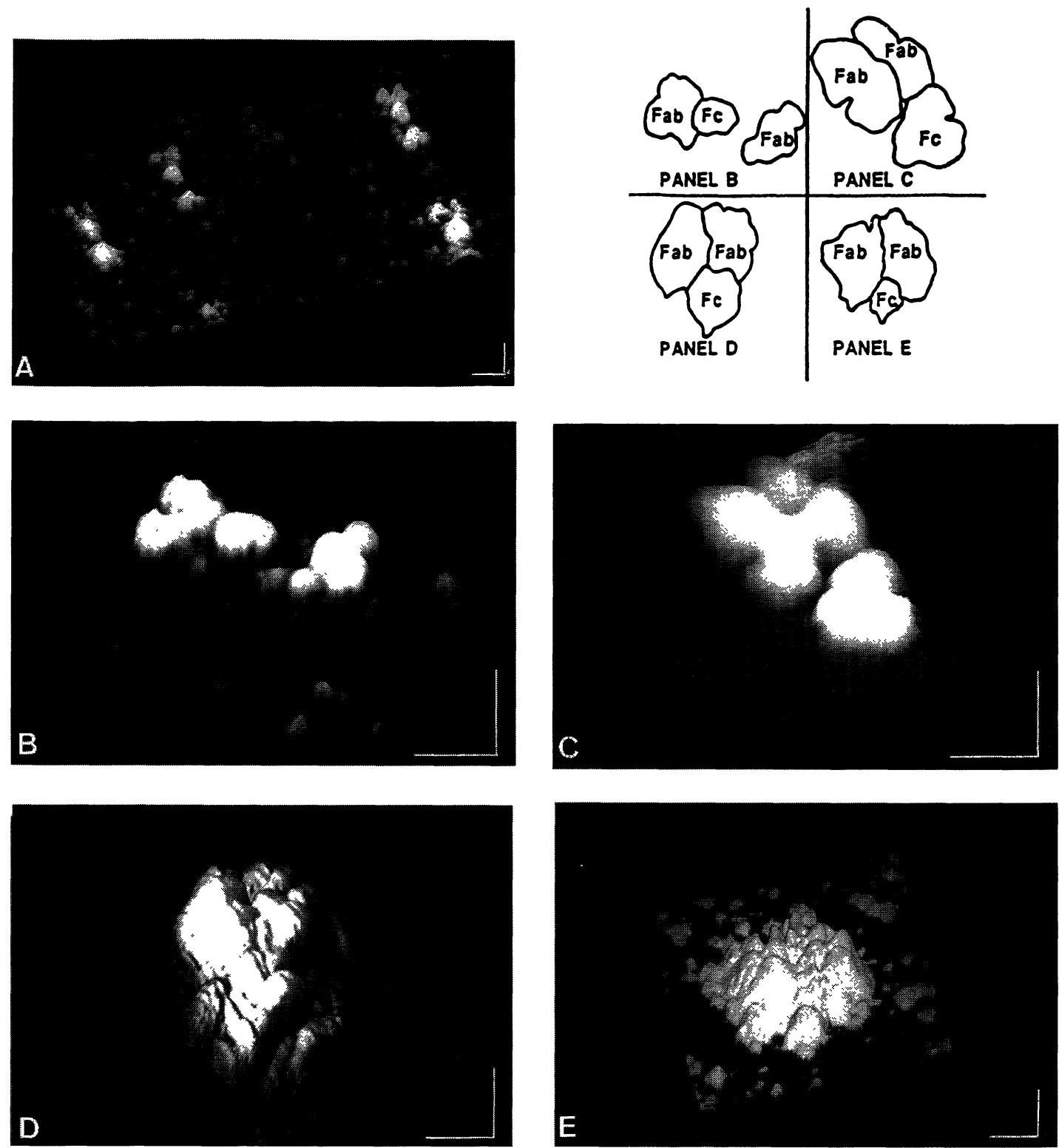

Fig. 1. - Pt/C-coated mouse monoclonal $\mathrm{IgG}_{1}$ on mica. The $\mathrm{IgG}_{1}$ solution $(3 \mu \mathrm{l}, 50 \mu \mathrm{g} / \mathrm{ml})$ was deposited onto graphite, dried and shadowed. The samples were then observed with the STM. Images obtained with four different samples are shown in pseudo-three-dimensional representation (panel A-C) and computer simulated light (panel D and E). The bars represent $100 \AA$. Panel A shows a $1330 \times 650 \AA$ frame with four similarly disposed structures which sizes correspond to an IgG molecule (a multiple-tip artifact cannot be excluded). Panel B is a close-up view of the upper right structure of panel A after a $90^{\circ}$ rotation in the plane. The two parts on each side are tentatively assigned to the Fab fragments and the one in the centre to the Fc fragment. Panel C shows another structure which may be interpreted as the image of an IgG molecule with the two Fab's almost parallel (size $150 \times 250 \AA$ ). In panel D, the image is similar but the parts corresponding to the Fab fragments form a $25^{\circ}$ angle and the Fc protruding towards the bottom of the picture (size: $215 \times$ $240 \AA)$. Panel E shows a structure reminiscent of those in panels A and B but of a larger size $(290 \times 315 \AA)$. Panels A, C, D and E correspond to independent experiments. 


\section{Results and discussion.}

Mica, an hydrophilic substrate allowing homogenous spreading of proteins, was coated by evaporation of a thin $\mathrm{Pt} / \mathrm{C}$ film. Conductivity of the $\mathrm{Pt} / \mathrm{C}$ film was sufficient to allow STM imaging at low tunneling current (below $150 \mathrm{pA}$ ). STM images demonstrated homogenous covering, flat over hundreds of nanometers, of grains (diameter: $43 \pm 10 \AA, 29$ measurements, corrugation: $7.5 \pm$ $2.0 \AA, 9$ measurements). Thus, we expected that proteins which are five to ten times larger than the grains would remain apparent under the Pt/C coating [18].

The monoclonal $\mathrm{IgG}_{1}$ was then deposited on mica, dried and coated with $\mathrm{Pt} / \mathrm{C}$. Several preparations were made and structures showing sizes (typically 150 by $250 \AA$ ) consistent with antibody molecules were reproducibly imaged. Figure 1 shows a few examples of such structures. An approximate two-fold symmetry is obvious in all images as expected for monomeric IgG. Sometimes the size of the structure (Fig. 1E: 290 by $315 \AA$ ) seemed too large for a single IgG molecule. However we have observed that, for objects of this size, convolution between the tip and the object, added to the $\mathrm{Pt} / \mathrm{C}$ coating, may increase significantly the lateral dimensions of the object observed with the STM, even though the calibration of the STM is essentially correct [18]. The presence of a proportion of aggregates of two IgG molecules is an alternative explanation. Apart for the size and the two-fold symmetry, the objects look different from each other which may be explained by a variable adsorption of the IgG molecule on mica and by the angle between the two Fab fragments which varies from an antibody molecule to an other in the same preparation, as demonstrated earlier by electron microscopy using negative staining [19]. By comparison, electron microscopy performed on carbon replicas of antibodies deposited on mica and rotary shadowed with $\mathrm{Pt} / \mathrm{C}$ at low angle showed a good density of molecules on the substrate but did not allow us to derive any information about the shape of the IgG molecule (Fig. 2).

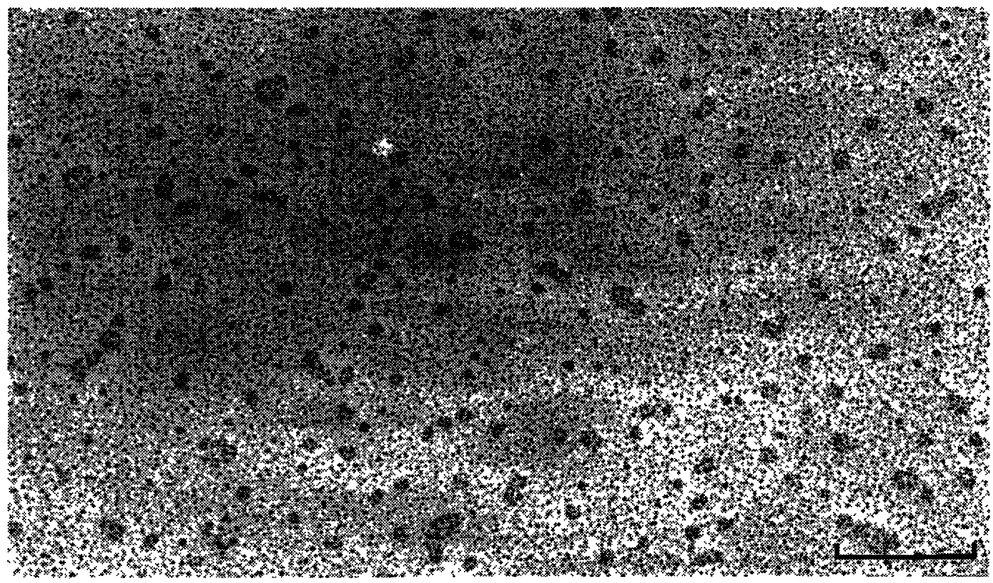

Fig. 2. - Electron microscopy of $\mathrm{IgG}_{1}$ molecules. The sample was prepared as above except that $\mathrm{Pt} / \mathrm{C}$ was rotary evaporated at low angle $\left(10^{\circ}\right)$ onto the sample followed by right angle evaporation of $\mathrm{C}$ alone. The sample was observed with a Philips EM 300 electron microscope. The bar is $2000 \AA$ long. Each grain spot corresponds to an $\mathrm{IgG}_{1}$ molecule. 

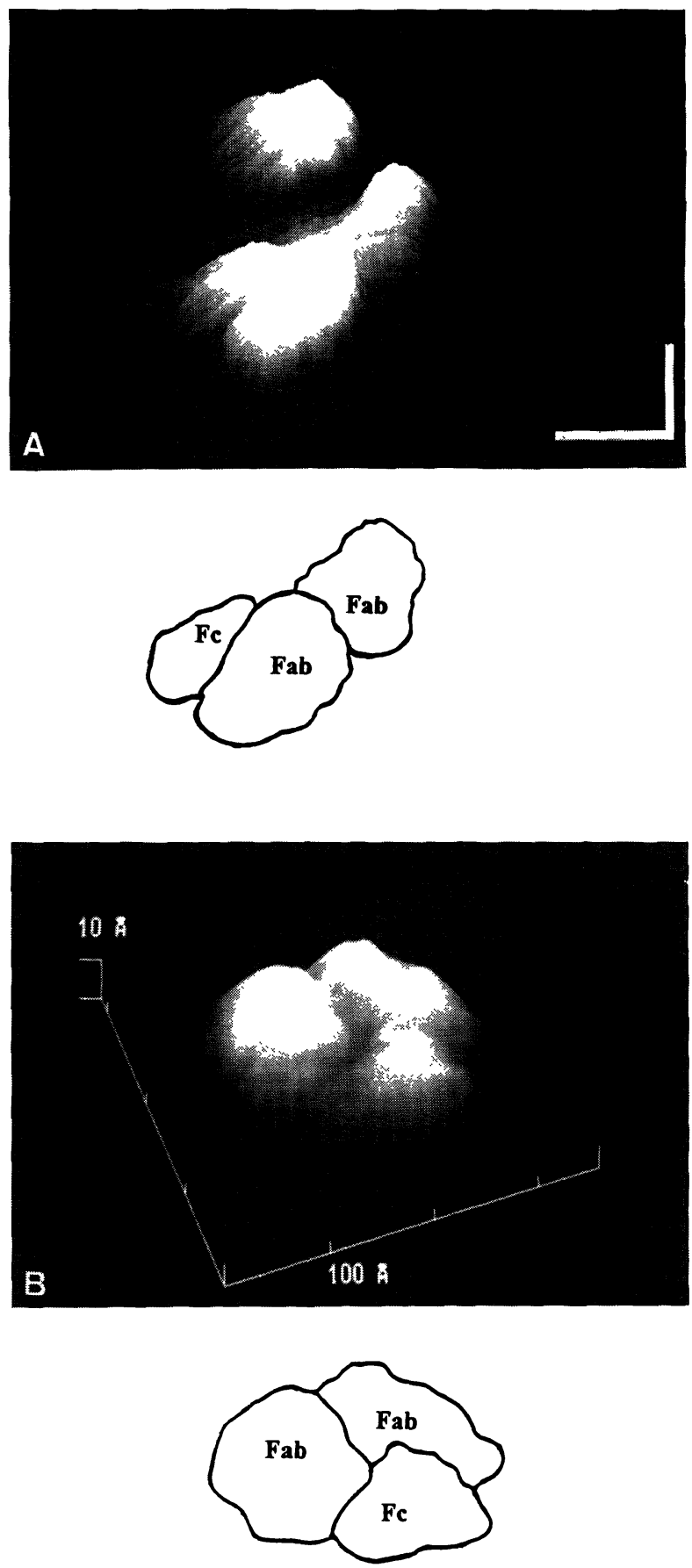

Fig. 3. - Uncoated mouse monoclonal $\operatorname{IgG}_{1}$ on graphite. The purified immunoglobulin solution was deposited on freshly cleaved HOPG, dried under vacuum, and imaged with the STM. Panel A: (tip bias: -200 $\mathrm{mV}$, tunneling current: $50 \mathrm{pA}$ ) the lower part of the figure shows a structure composed of two parts on the right which measure 80 by $40 \AA$ (the expected size for Fab fragments) and one part on the left which may correspond to the Fc fragment. The size of this three-domain structure is approximately $250 \times 150 \AA$. The isolated structure at the top of the figure may be a contaminant. Panel B: (tip bias: $-600 \mathrm{mV}$, tunneling current $4 \mathrm{pA})$ the molecule appears as expected as a three domain structure whose dimensions $(255 \times 206$ $\AA$ ) agree closely with the expected size of an IgG. Panels A and B correspond to independent experiments. 

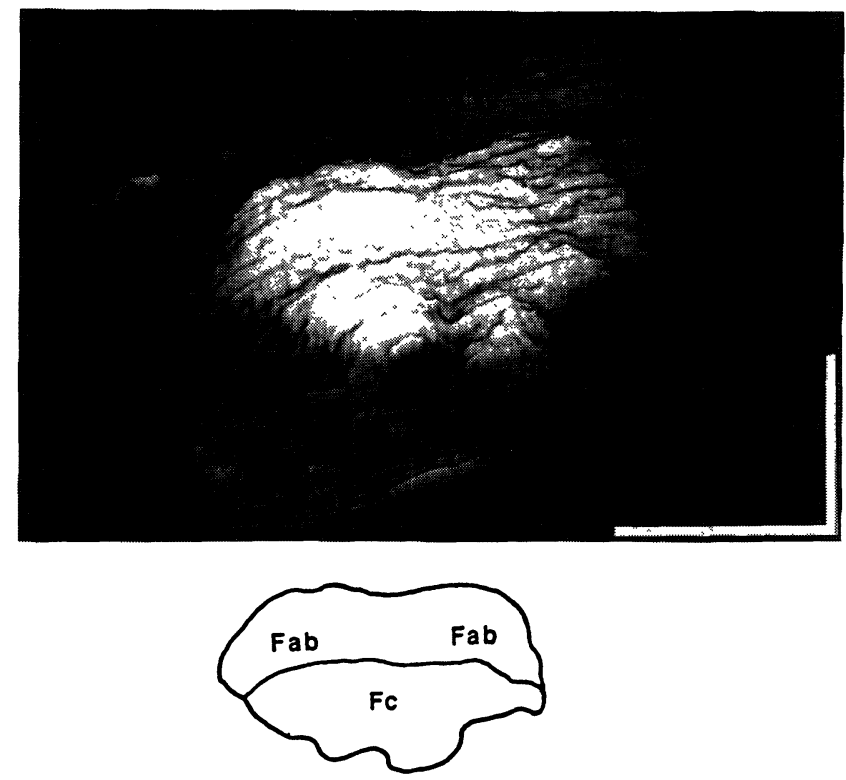

Fig. 4. - Uncoated mouse monoclonal $\mathrm{IgG}_{1}$ on glow discharge treated graphite. HOPG was treated by glow discharge for $10 \mathrm{sec}$ in air at 0.1 Torr and the IgG solution was deposited immediately after. The sample was imaged after drying under vacuum $(0.01$ Torr $)$. The largest upper part $(190 \times 60 \AA)$ may represent the image of the two Fab fragments packed together, the lower triangular part $(100 \times 70 \AA)$ corresponding to the Fc fragment.

We then imaged the uncoated immunoglobulin $\mathrm{G}$ deposited on highly oriented pyrolytic graphite (HOPG). Depending on the concentrations of IgG used and on the imaging conditions we observed either flat areas of apparently bare graphite with occasional steps and images similar to those described by Clemmer and Beebe [20], or large aggregates without apparent structure that changed from scan to scan. In several occasions, however, we imaged objects like the one shown in figure 3, panel A, clearly composed of two similar structures $(80 \times 40 \AA)$ resembling the Fab fragments of an IgG associated with a lower part whose sides measure $60 \AA$ interpreted as the Fc fragment. The slight asymmetry of the image compares with that found in the recently published crystallographic study of an IgG with a normal Hinge region [21]. The apparent height of the structure shown in the figure is too small for an IgG (less than $10 \AA$ ). However, for materials with poor conductivity, this measurement is not reliable [22]. Other structures, like the one seen on the top of the figure, remain to be explained and may be contaminants, although the IgG preparation was essentially pure (see Materials and Methods). As low tunneling currents (50 pA) appeared useful to obtain stable images, we attempted imaging using even lower tunneling currents. Interesting images of an IgG antibody and of the complexes between the antibody and its antigen, the Thy-1 molecule [23] were obtained using a tunneling current of $30 \mathrm{pA}$ (not shown). In figure 3 , panel B, we show an image of an IgG molecule recorded using a tunneling current of only $4 \mathrm{pA}$. The overall shape of the molecule is conserved and the lateral sizes agree with the expected size of an IgG. The image is even closer to the present models [24] and to the crystal structure of intact $\operatorname{IgG}[21]$ and the measured vertical size is close to $16 \AA$.

Imaging an isolated molecule passively adsorbed onto the hydrophobic and extremely flat surface of graphite may be made difficult by the weak interaction between IgG, an hydrophilic protein, and the substratum. We thus submitted the surface to glow discharge. We observed that prolonged discharges ( $>30 \mathrm{~s}$ ) transformed the flat surface of HOPG into grains, rather homogenous 


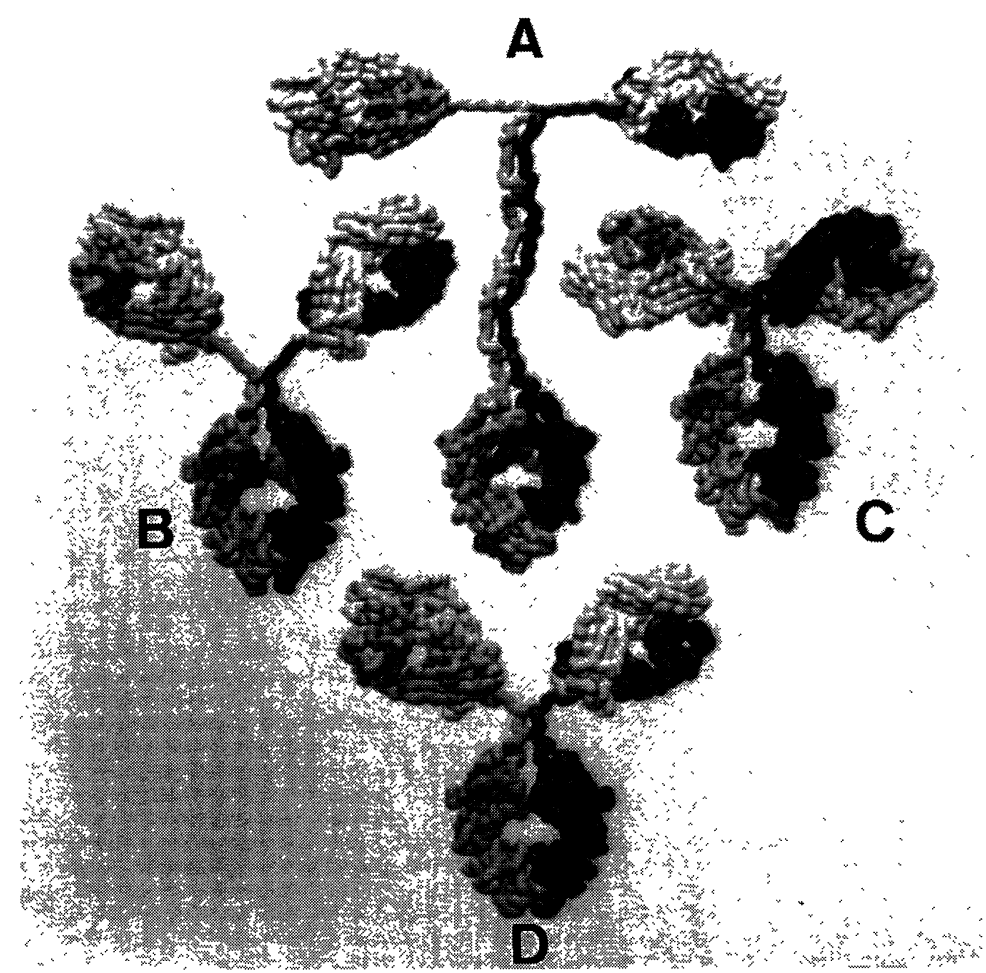

Fig. 5. - Computer models of the four human IgG subclasses. The pictures were produced using the program IMMAM by R.H.S. Pumphrey and reproduced with permission (courtesy of the Computer Graphics Unit, University of Manchester. A) $\left.\left.\mathrm{IgG}_{3}, \mathrm{~B}\right) \mathrm{IgG}_{1}, \mathrm{C}\right) \mathrm{IgG}_{2}$, D) $\mathrm{IgG}_{4}$ ).

in size but probably too large to allow us to distinguish adsorbed molecules from the background. Water and aqueous salt solutions were spreading freely onto the treated graphite, indicating that the surface had been made hydrophilic. Shorter discharges (10 to $30 \mathrm{~s})$ induced less corrugation that may be compatible with STM imaging of adsorbed material and deposition of water droplets gave variable results, from none to complete wetting.

We deposited IgG solutions in ammonium acetate on graphite treated with short discharges. STM imaging showed relatively small corrugation and some structures were apparent. We found a few candidates for the image of an IgG molecule whose best example is shown in figure 4. Again, the size and approximate symmetry are present. The height of the structure (about $30 \AA$ ) is compatible with that of an IgG molecule. Details are present in the image. However they changed slightly from scan to scan and the small number of images obtained makes further discussion premature. Other reports showing images of antibodies also illustrate the difficulty to obtain reliable structural information with uncoated globular proteins using the STM $[25,26]$ or the Atomic Force Microscope [27].

\section{Conclusion.}

Figure 5 shows models of the IgG three-dimensional structure for comparison with the STM images. We consider that the images reported in this paper agree with the general sizes and shapes that may be infered from these models. The next step in STM imaging will be to image molecules 
of unknown three-dimensional structure. This has been attempted recently [23]. A great deal of further experimental work is still needed to make the STM a routine instrument for the study of protein structure. Many avenues are being explored. We have tried three of them: metal shadowing after deposition on a favorable substratum (mica), deposition on graphite and very low tunneling currents and deposition on a conducting substratum (graphite) whose adsorption properties were improved by glow discharge. Metal coating, because of its reproducibility, will allow us to address questions about interactions between relatively large molecules. For uncoated proteins, the interesting observation that the height of the observed molecules is increased by both the decrease of the tunneling current intensity and the glow discharge treatment is possibly indicative of the role of the electron transfer rate - in the molecule and between the molecule and the substrate - in the imaging process. Direct observation, through progress in the preparation of adequate substratum, in the quality of the tips and in the performance of the STM (lower tunneling current, scanning speed, stability), will make possible predictions about proteins of unknown three- dimensional structure.

\section{Acknowledgements.}

This work was sponsored by the CNRS. It also received financial support from INSERM, the University of Aix-Marseille II, the Faculté des Sciences de Luminy, the Mairie de Marseille, the Conseil Général des Bouches du Rhône, and the Conseil Régional de la Région Provence-AlpesCôte d'Azur. The authors are very grateful to Alain Humbert and his colleagues for sharing their experience of scanning tunneling microscopy and for the frequent and helpful discussions.

\section{References}

[1] Binnig G. and Rohrer H., Helv. Phys. Acta 55 (1982) 726.

[2] Binnig G., Rohrer H., Gerber C. and Weibel E., Phys. Rev. Lett. 57 (1983) 120.

[3] Beebe T.P. Jr, Wilson T.E., Ogletree D.F., Katz J.E., Balhorn R., Salmeron L.B. and Siekhaus W.J., Science 243 (1989) 370.

[4] Driscoll R.J., Youngquist M.G. and Baldeschwieler J.D., Nature 346 (1990) 294.

[5] Dunlap D. and Bustamante C., Nature 342 (1989) 204.

[6] Amrein M., Stasiak A., Gross H., Stoll E. and Travaglini G., Science 240 (1988) 514.

[7] Amrein M., Dürr R., Stasiak A., Gross H. and Travaglini G., Science 243 (1989) 1708.

[8] Welland M.E., Miles M.J., Lambert N., Morris V.J., Coombs J.H. and Pethica J.B., Int. J. Biol. Macromol. 11 (1989) 29.

[9] Yang J., Takeyasu K., Somlyo A.P. and Shao Z., Ultramicroscopy 45 (1992) 199.

[10] Haggerty L. and Lenhoff A.M., Biophys J. 64 (1993) 886.

[11] Leggett G.J., Roberts C.J., Williams P.M., Davies M.C., Jackson D.E. and Tendler S.J.B., Langmuir 9 (1993) 2356.

[12] Cooper J.M., Shen J., Yound F.M., Connolly P. and Barker J.R., J. Mater. Sci. 5 (1994) 106.

[13] Kreusel K.M., Adair J.R., Beeley N.R.A., Davies M.C., Jackson D.E., Roberts C.J., Tendler S.J.B. and Williams P.M., J. Vac. Sci. Technol. B 12 (1994) 1517.

[14] Guckenberger R., Wiegräbe W. and Baumeister W., J. Microscopy 152 (1988) 795.

[15] Guckenberger R., Wiegräbe W., Hillebrand A., Hartmann T., Wang Z. and Baumeister W., Ultramicroscopy 31 (1989) 327.

[16] Hood L., Kronenberg M. and Hunkapiller T., Cell 40 (1985) 225.

[17] Humbert A., Dayez M., Granjeaud S. and Ricci P., J. Vac. Sci. Technol. B9 (1991) 804.

[18] Barbet J., Garvin A., Thimonier J., Chauvin J.P. and Rocca-Serra J., Ultramicroscopy 50 (1993) 355.

[19] Roux K.H. and Metzger D.W., J. Immunol. 129 (1982) 2548. 
[20] Clemmer C.R. and Beebe T.P. Jr, Science 251 (1991) 640.

[21] Harris L.J., Larson S.B., Hasel K.W., Day J., Greenwood A. and McPherson A., Nature 360 (1992) 369.

[22] Binnig G. and Rohrer H., in: Trends in Physics 1984, J. Janta and J. Pantoflicek Eds. (European Physical Society, The Hague, 1984) 38.

[23] Rocca-Serra J., Thimonier J., Chauvin J.P. and Barbet J., J. Vac. Sci. Technol. B 12 (1994) 1490.

[24] Pumphrey R.H.S., in: The human IgG subclasses, F. Shakib Ed. (Pergamon press, London, 1986).

[25] Olk C.H., Heremans J., Lee P.S., Dziedic D. and Sargent N.E., J. Vac. Sci. Technol. B9 (1991) 1268.

[26] Leatherbarrow R.J., Stedman M. and Wells T.N.C., J. Mol. Biol. 221 (1991) 361.

[27] Weisenhorn A.L., Gaub H.E., Hansma H.G., Sinsheimer R.L., Kelderman G.L. and Hansma P.K., Scanning Microscopy 4 (1990) 511. 